\title{
ERRATUM
}

\section{Cystemustine in recurrent high grade glioma}

\author{
X. Durando $\cdot$ E. Thivat $\cdot$ H. Roché $\cdot$ J. O. Bay $\cdot$ \\ J.-J. Lemaire $\cdot$ P. Verrelle $\cdot$ J. Chazal $\cdot$ \\ H. Curé · P. Chollet
}

Published online: 23 June 2006

(C) Springer Science+Business Media B.V. 2006

Journal of Neuro-Oncology

DOI 10.1007/s11060-005-9096-7

As the contents of our publication were not reviewed nor approved by the EORTC and since M.-A. Lentz has not directly participated in redaction of this article, her name is deleted from the authors' list.

Moreover, we would like to add an acknowledgement to the EORTC-CSG/ECSG group for having given us access to the data of their phase II clinical trial.

The online version of the original article can be found at http://dx.doi.org/10.1007/s11060-005-9096-7

X. Durando $\cdot$ E. Thivat $\cdot$ J. O. Bay $\cdot$ P. Verrelle

H. Curé · P. Chollet

Centre Jean Perrin, 63011, Clermont-Ferrand Cédex,

France

E. Thivat $\cdot$ P. Chollet

Inserm, U484, Rue Montalembert, 63011,

Clermont-Ferrand, France

H. Roché

Centre Claudius Regaud, 20-24 rue du Pont Saint-Pierre,

31052, Toulouse Cédex, France

J.-J. Lemaire · J. Chazal

CHU Hopital Gabriel Montpied, Clermont-Ferrand, France

X. Durando $(\square)$

Centre Jean Perrin, 58 Rue Montalembert, BP 392, 63011,

Clermont-Ferrand Cédex, France

e-mail: Recherche.CLINIQUE@cjp.fr 